\title{
SEARCHES FOR EXOTIC PHENOMENA WITH THE ATLAS DETECTOR*
}

\author{
NeKtarios Ch. Benekos \\ on behalf of the ATLAS Collaboration \\ National Technical University of Athens, Athens, Greece
}

(Received February 20, 2018)

\begin{abstract}
The significant increase of the centre-of-mass energy of the Large Hadron Collider (LHC) from 8 to $13 \mathrm{TeV}$ has allowed the LHC experiments to explore previously inaccessible kinematic regimes in their search for phenomena beyond the Standard Model (BSM). Many BSM theories predict new phenomena accessible by the LHC. Searches for new physics models are performed using the ATLAS experiment at the LHC. The results reported here use the proton-proton $(p p)$ collision data sample collected in 2015 and 2016 by the ATLAS detector at the LHC with a centre-of-mass energy of $13 \mathrm{TeV}$ with a total integrated luminosity of $45 \mathrm{fb}^{-1}$.
\end{abstract}

DOI:10.5506/APhysPolBSupp.11.419

\section{Introduction}

Since its formulation, the Standard Model (SM) of particle physics has enjoyed unblemished experimental success. However, the theory leaves certain structural questions open: Why are there three generations of quarks and leptons? What is behind the observed mass hierarchy? What is the nature of dark matter? As well as more experimentally driven questions about the nature of dark matter.

A plethora of extensions to the SM, known as Exotic models, have been developed in order to address these questions. Many of these models indicate that new phenomena are likely to appear at the $\mathrm{TeV}$-scale, the direct probe of which is possible with the ATLAS detector [1] at the LHC [2] at CERN. The ATLAS detector is a multi-purpose detector whose tracking, calorimetric, and muon spectrometer systems allow accurate identification and precise reconstruction of leptons, jets, photons, and missing transverse

* Presented at "Excited QCD 2018", Kopaonik, Serbia, March 11-15, 2018. 
energy $\left(E_{\mathrm{T}}^{\mathrm{miss}}\right)$. In the analyses summarized in this note, the theoretical frameworks considered are: extra dimensions such as ADD, Quantum Black Hole (QBH), Randall-Sundrum (RS), new gauge boson (Sequential Standard Model (SSM), leptophobic-Z', Heavy Vector Triplet (HVT)), contact interaction (CI), excited fermions. The considered final states include vector bosons, jets, leptons and photons.

\section{Searching for new gauge bosons}

Multiple theories beyond the SM postulate the existence of extra gauge bosons, which can be probed in various channels.

Searching for a $W^{\prime}$ heavy boson: The search is conducted in the $W^{\prime} \rightarrow \ell \nu$ channel, where $\ell=e$ or $\mu$. The signature is a single high- $p_{\mathrm{T}}$ isolated lepton and substantial missing transverse energy $E_{\mathrm{T}}^{\text {miss }}$ due to the undetected neutrino. The signal discriminant is the transverse mass of the lepton and neutrino, $m_{\mathrm{T}}=\sqrt{2 p_{\mathrm{T}} E_{\mathrm{T}}^{\mathrm{miss}}\left(1-\cos \varphi_{\ell \nu}\right)}$, which is compared to the expectation from SM processes. A good agreement is observed, as shown in Fig. 1 (left). The resulting limits on the cross section times branching ratio $(\sigma \times \mathrm{BR})$ for a $W^{\prime}$ boson as a function of mass are shown in Fig. 1 (right). The results of this search [3] are interpreted in the context of the benchmark
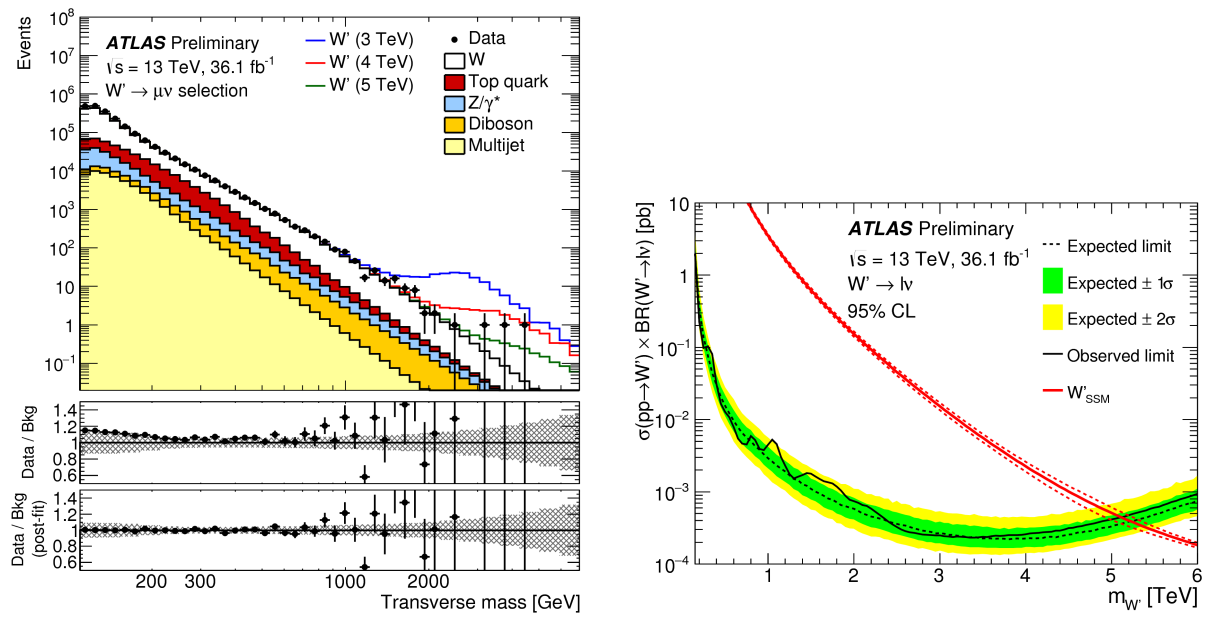

Fig. 1. (Colour on-line) Left: Transverse mass distributions for events satisfying all selection criteria in the muon channel. The distributions are compared to the sum of all expected backgrounds, with three selected $W_{\mathrm{SSM}}^{\prime}$ signals overlaid. The band in the ratio plot shows the systematic uncertainty [3]. Right: Median expected (dashed black line) and observed (solid black line) 95\% confidence level (C.L.) upper limits on $\sigma \times \mathrm{BR}$. The predicted $\sigma \times \mathrm{BR}$ cross section for $W_{\mathrm{SSM}}^{\prime}$ production is shown as a solid grey (red) line [3]. 
Sequential Standard Model (SSM) [4] where the couplings of the $W^{\prime}$ to fermions are assumed to be identical to those of the SM $W$. Interference between the SSM $W^{\prime}$ and the SM $W$ and the decay into other bosons is neglected.

Search for high-mass opposite-sign dilepton resonances: The $\ell^{+} \ell^{-}$final-state signature is a key search channel for a host of different new phenomena expected in theories that go beyond the SM. The $\ell^{+} \ell^{-}$ invariant mass $\left(m_{\ell \ell}\right)$ distribution could deviate from the SM expectation due either to the presence of a $Z^{\prime}$ resonance [5] or the presence of new nonresonant couplings [6]. A search for these effects was performed in both the electron and muon channels, with the $m_{\ell \ell}$ distributions compared to the SM prediction [7]. This search channel benefits from high signal selection efficiencies and relatively small, well-understood backgrounds. No significant deviations are observed, and the resulting limits on the $\sigma \times \mathrm{BR}$ for a $Z^{\prime}$ boson as a function of mass are shown in Fig. 2 (left). The lower 95\% C.L. exclusion limits on the energy scale $\Lambda$ for various $\ell \ell q q$ contact interaction models range between $23.5 \mathrm{TeV}$ and $40.1 \mathrm{TeV}$, Fig. 2 (right).
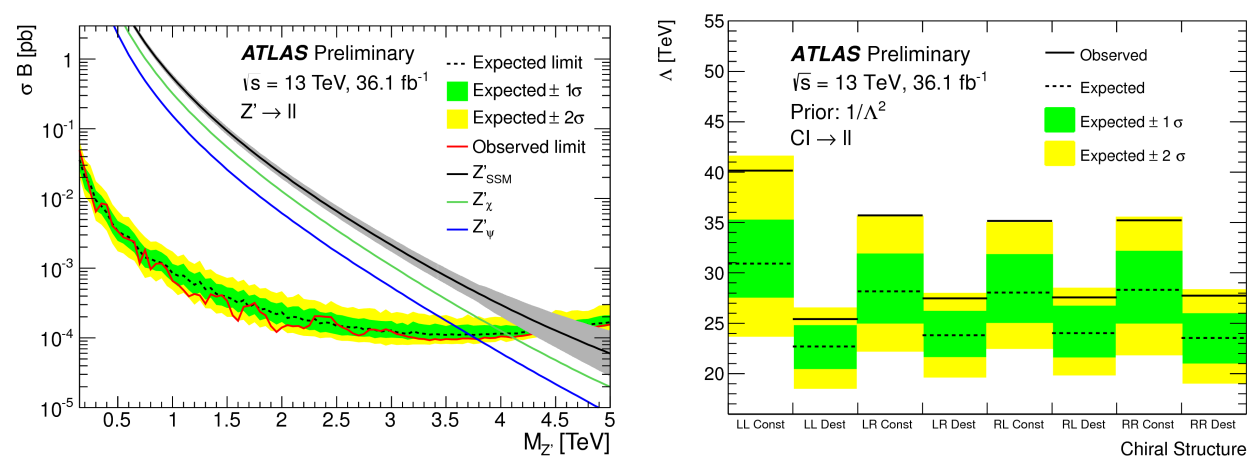

Fig. 2. Left: Upper 95\% C.L. limits for $Z^{\prime}$ production $\sigma \times$ BR to two leptons. Right: Lower $95 \%$ C.L. limits on the contact interaction (CI) scale $\Lambda$ for different chiral coupling and both constructive and destructive interference scenarios using a uniform positive prior in $1 / \Lambda^{2}$.

Searches for the same-sign dilepton final states: Events with two high- $p_{\mathrm{T}}$, isolated, prompt leptons with the same electric charge (samesign leptons) are produced very rarely in a $p p$ collision according to the predictions of the SM, but they may occur with a higher rate in various beyond the SM theories. This analysis aims to study BSM theories that contain a doubly-charged Higgs particle [8,9], and in the absence of evidence for a signal, set limits through the observed invariant mass of the samesign leptons. A model-independent search for new physics processes was performed and no clear evidence for an excess in the observed same-sign 
electron pair invariant mass spectrum was observed within systematic and statistical uncertainties. Therefore, the upper limits for the signal cross sections were set at $95 \%$ C.L. [10].

Searches for the opposite-sign different flavor $(e \mu)$ dilepton final states: Within the SM, direct production of lepton pairs with different flavour is forbidden due to lepton flavour conservation (LFC). However, lepton flavour violation (LFV) is allowed in many extensions of the SM such as ADD [11] and RS [12] models for extra dimensions. A search for such resonance was carried out by comparing the $e \mu$ invariant mass distribution to the SM expectation [13]. A good agreement is observed, and the resulting limits on the $\sigma \times \mathrm{BR}$ for a LFV $Z^{\prime}$ as a function of the $Z^{\prime}$ mass are shown in Fig. 3.
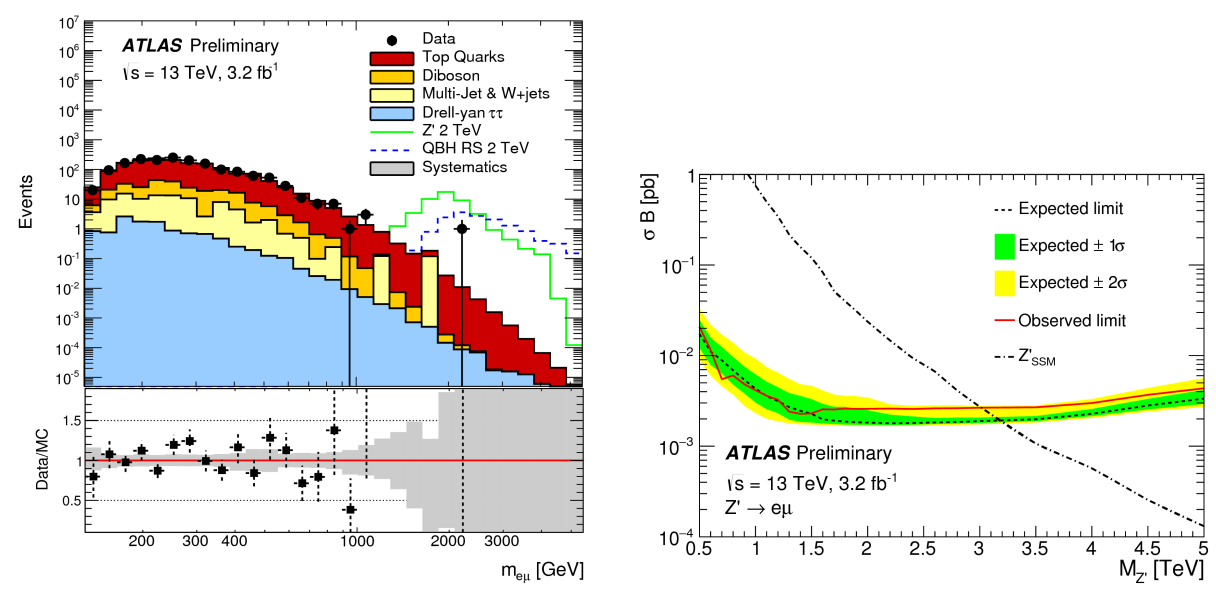

Fig. 3. Left: The invariant mass distribution of selected electron-muon pairs for data and MC expectation. The errors show the statistical uncertainty on the observed yields, while the systematic band includes the addition in quadrature of all systematic uncertainties. Right: The expected and observed 95\% C.L. lower mass limits on the $Z^{\prime}$ production cross section in decays to an $e \mu$ final state [13].

\section{Searches for $\gamma \gamma$ resonances}

The diphoton $(\gamma \gamma)$ channel is very promising since it is a very clean channel, requiring just two energetic isolated prompt photons in the final state. Two analyses are optimized, with different cuts on isolation and on the $p_{\mathrm{T}}$ of the photons, for the search of a scalar Higgs-like boson [14] and for the search of spin-2 graviton [15]. Analyzing the combined 2015-2016 data [14] corresponding to $15.4 \mathrm{fb}^{-1}$ of total integrated luminosity, no evident excess was found around $750 \mathrm{GeV}$ for the spin-0 analysis (as seen in Fig. 4 (right)). The largest local significance is $2.4 \sigma$ for a mass near $1600 \mathrm{GeV}$ and a narrow 
width hypothesis. In the $700-800 \mathrm{GeV}$ mass range, the largest local significance is $2.3 \sigma$ for a mass near $710 \mathrm{GeV}$ and a relative width of $10 \%$. The corresponding global significance of excess of events is less that $1 \sigma$. Looking at the analysis performed with the 2015-2016 collected events, the most probable explanation is that the excess around $750 \mathrm{GeV}$ found in $3.2 \mathrm{fb}^{-1}$ of 2015 data [15] was a statistical fluctuation.
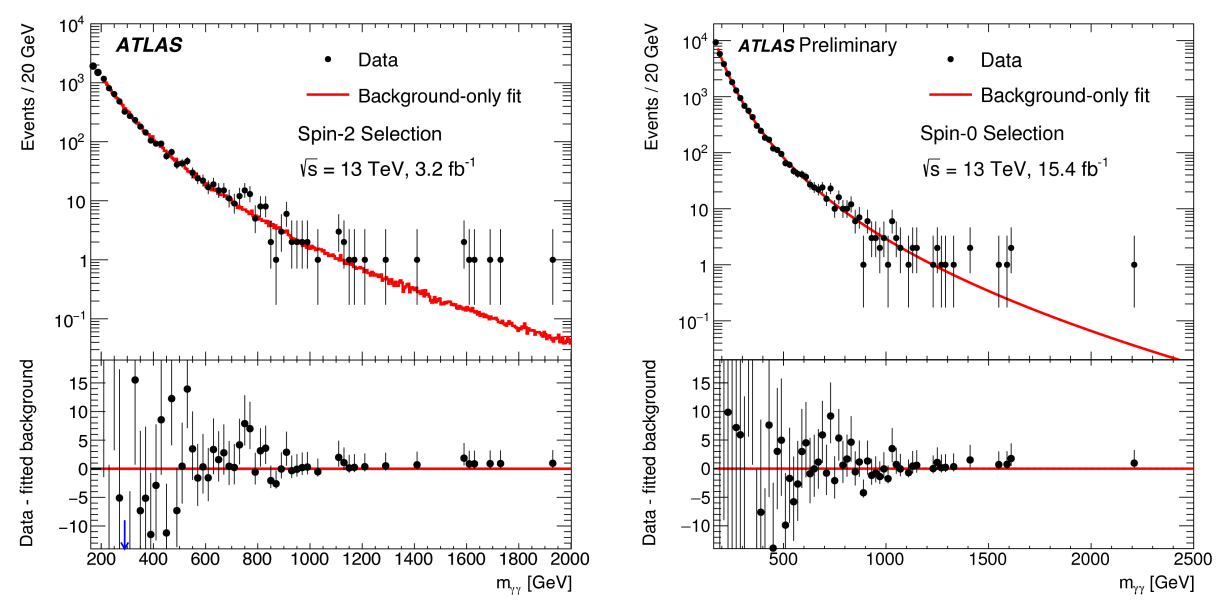

Fig. 4. Distribution of the diphoton invariant mass for the selection used in the search for a spin-2 (left) [15] and spin-0 (right) [14] resonance with the best background-only fit.

\section{Diboson resonance searches}

Several models can be tested with diboson final states $(W W, W Z, Z Z)$, including heavy CP-even scalar singlet $(X \rightarrow W W / Z Z)$, Heavy Vector Triplet $\left(W^{\prime} \rightarrow W Z, Z^{\prime} \rightarrow W W\right)$ and $\mathrm{RS}$ spin-2 graviton $(G \rightarrow W W / Z Z)$. Both leptonic and hadronic decays of the vector boson are used in these searches. Given the presence of SM $W / Z / \gamma$ boson pairs in SM productions and decay products predicted in various theory models/mechanisms, the searches have quite some diversities in terms of both the decay final states of the new physics interpreted resonance and the further decay final states of various SM bosons. At high-energy, the reconstruction of boosted bosons is difficult due to the fact that the products of the decay are very collimated. In the case of the hadronic decay, the two jets originating from the two quarks are reconstructed as a single large-Radius (large- $R$ ) jet and a jet substructure technique has been developed to extract the SM $W / Z$ boson decayed hadrons out from the enormous QCD multi-jet backgrounds. The technique is deployed in all the hadron decay final-state analysis of massive diboson resonance searches (including semi-leptonic $W / Z+\gamma$ but excluding $\gamma \gamma$ ). 
One of the experimental hotspot in ATLAS Run 1 (with $8 \mathrm{TeV}$ data) diboson results is the $2 \mathrm{TeV}$ excess found in di-large- $R$-jet searches, which has $3.4 \sigma$ local significance (2.5 $\sigma$ including LEE) [16]. The excess was very well investigated in ATLAS Run 2 with the combined 2015 (full) +2016 (partial) dataset, with the expected sensitivity competitive with full 2012 Run 1 dataset. The excess is revisited in $13 \mathrm{TeV}$ data and no equally evident excess is observed [17], which stays tuned as long as more data is accumulating.

The other decay channels such as $W(\rightarrow e \nu) V(\rightarrow q q)$ and $Z(\rightarrow \ell \bar{\ell} / \nu \bar{\nu})$ $V(\rightarrow q q)$ are also intensively analyzed and the results are presented with the same dataset at $13 \mathrm{TeV}$. The invariant mass spectra of various diboson channels are summarized in Fig. 5 and the limits on the cross section of new physics decaying into $W Z$ are summarized in Fig. 6, taking the HVT $W^{\prime} \rightarrow W Z$ as the benchmark model which is excluded up to $2.4 \mathrm{TeV}$ at 95\% C.L. [18-20].
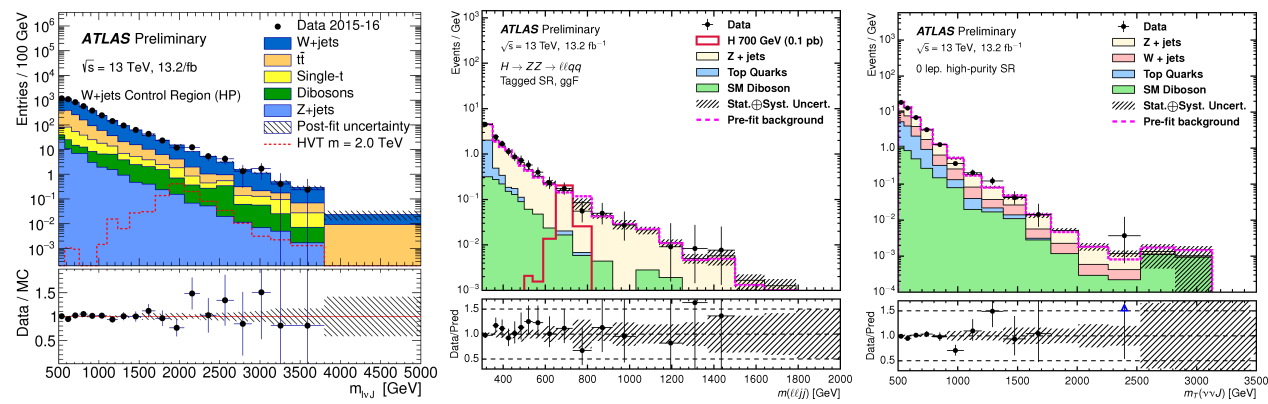

Fig. 5. The diboson invariant mass distribution in $W(\rightarrow e \nu) V(\rightarrow q q)$ (left), $Z(\rightarrow \ell \bar{\ell}) V(\rightarrow q q)$ (middle) and $Z(\rightarrow \nu \bar{\nu}) V(\rightarrow q q)$ (right) final states using $13 \mathrm{TeV}$ $2015+2016$ combined dataset by ATLAS, with the ratio of the DATA/MC shown at the lower panel $[18,19]$.

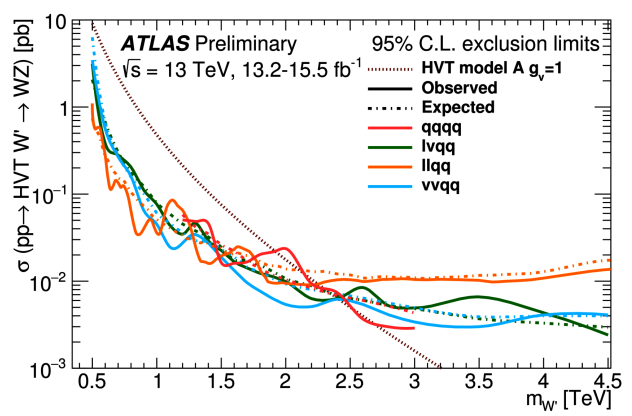

Fig. 6. The summary of the current $X \rightarrow$ diboson cross-section limits in comparison with HVT $W^{\prime} \rightarrow W Z$ benchmark model [20]. 


\section{The dijet resonace searches}

A search for new physics via a jet-pair (dijets) final state is performed by ATLAS at $\sqrt{s}=13 \mathrm{TeV}$, using $2015\left(3.5 \mathrm{fb}^{-1}\right)$ and $2016\left(33.5 \mathrm{fb}^{-1}\right) \mathrm{com}-$ bined dataset of $p p$ collisions. Mass and angular distributions of dijet events are taken as the observables to examine the new physics anomaly signatures. No significant deviations or excesses are found in the observed angular distributions and dijet mass spectra, in comparison with the SM. Invariant mass and angular distributions are compared to background predictions and no significant deviation is observed. For resonance searches, a new method for fitting the background component of the invariant mass distribution is employed. The dataset is then used to set upper limits at a 95\% C.L. on a range of new physics scenarios. Excited quarks with masses below $6.0 \mathrm{TeV}$ are excluded, and limits are set on quantum black holes, heavy $W^{\prime}$ bosons, $W^{*}$ bosons, and a range of masses and couplings in a $Z^{\prime}$ dark matter mediator model. Model-independent limits on signals with a Gaussian shape are also set, using a new approach allowing factorization of physics and detector effects. From the angular distributions, a scale of new physics in contact interaction models is excluded for scenarios with either constructive or destructive interference. These results represent a substantial improvement over those obtained previously with lower integrated luminosity [21]. Figure 7 shows the $95 \%$ C.L. upper limits obtained from the $m_{j j}$ distribution on $\sigma \times A$, for the chosen models.
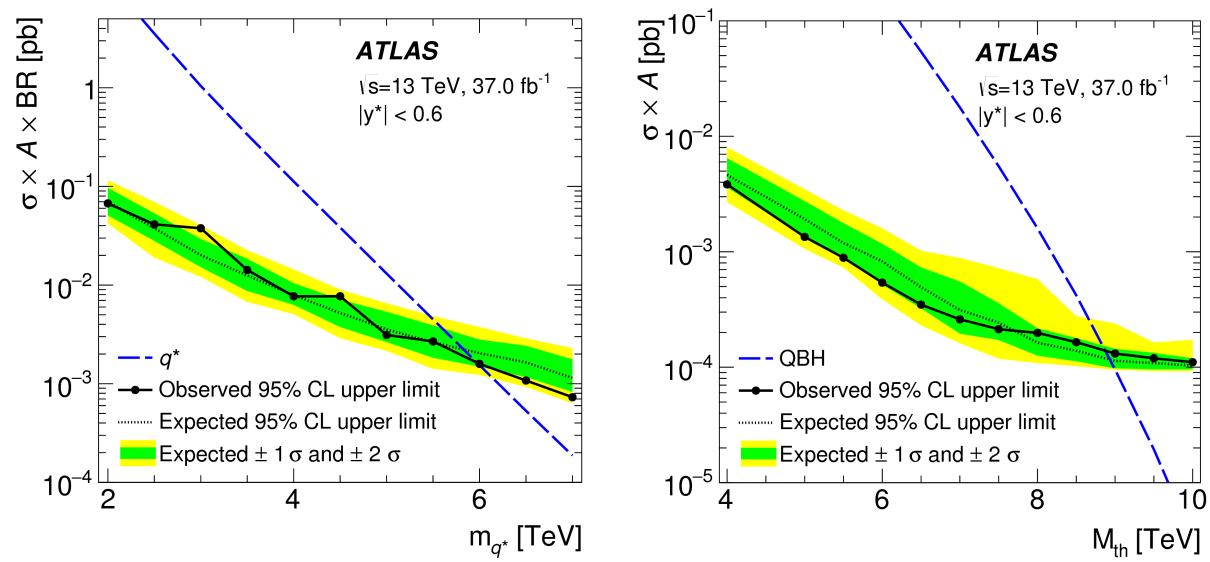

Fig. 7. 95\% C.L. upper limits obtained from the $m_{j j}$ distribution on cross section times acceptance $(\sigma \times A)$, for the chosen models: $q^{*}$ (left), quantum black holes with $n=6$ generated with BlackMax (right) [22]. 


\section{Dark matter searches}

Dark matter (DM) comprises approximately $27 \%$ of the universe, yet little is known about its properties. DM particles, if produced by the LHC, will leave a distinct signature of significant missing transverse momentum $\left(E_{\mathrm{T}}^{\mathrm{miss}}\right)$. ATLAS DM searches revolve around two main strategies [23]. The first approach is the search for events in which DM is pair-produced in association with another object, typically through the initial-state radiation (ISR). This ISR object is necessary, as the production of DM without other particle(s) is invisible to the detector, while visible object(s) plus DM will appear as a significant imbalance in the $E_{\mathrm{T}}^{\text {miss }}$ of the event. This class of searches is often referred to as $E_{\mathrm{T}}^{\mathrm{miss}}+X$. The $X$ can be interpreted to be QCD jets, SM vector boson $W / Z / \gamma$, the Higgs boson or even the quarkonia of heavy quarks such as $t \bar{t}$ and $b \bar{b}$.

The second approach is the search for mediators which could connect the SM to the DM. This strategy is useful as any SM process that can form the dark mediator can also decay back to the input SM particles, so long as the mediator is sufficiently massive. The most common example is the search for dijet resonances, where it is assumed that the mediator couples to light quarks or gluons, and an observed resonance could be interpreted as the production of a new dark mediator. In interpreting results from searches for DM or dark mediators, ATLAS primarily relies on simplified models connecting the two SM inputs to the two DM outputs via a single vector, axial vector, or scalar-mediator. Using such a simplified model means that DM and dark mediator searches are directly comparable. The ATLAS experiment provides the good platform for new physics search of DM given the nice performance of genuine lepton/photon identification, $E_{\mathrm{T}}^{\text {miss }}$ reconstruction and boson tagging. The summary of the current exclusion limits on DM mass-mediator mass is presented in Fig. 8.
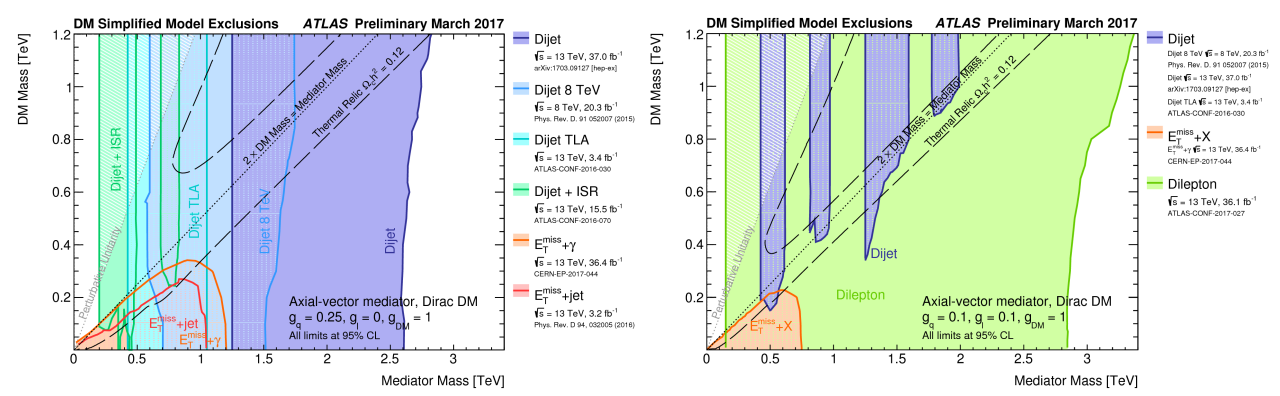

Fig. 8. A summary of ATLAS limits on the leptophobic axial vector mediators coupling to DM, with variable mediator and DM masses, from both the leading $E_{\mathrm{T}}^{\text {miss }}+X$ analyses and dark mediator searches. Coupling values are fixed to 0.25 for quarks and 1 for DM (left) or 0.1 for quarks and 1 for DM (right) [20]. 


\section{Conclusion}

We present a summary of the latest exotic new physics search results in ATLAS experiment using $\sqrt{s}=13 \mathrm{TeV} p p$ collision data recorded in years 2015 and 2016 by the ATLAS experiment at the LHC. The search for new phenomena beyond the SM is a very active field at the ATLAS experiment, of which only a subset of results is presented here. No evidence of new physics was revealed in these searches. The search results provide the most up-to-date and stringent constraints on many new physics models and frameworks never tested by any other experiment. An overall summary of the current Exotic results are presented in [20].

\section{REFERENCES}

[1] G. Aad et al., JINST 3, S08003 (2008).

[2] L. Evans, P. Bryant, JINST 3, S08001 (2008).

[3] ATLAS Collaboration, ATLAS-CONF-2017-016, 2017.

[4] G. Altarelli, B. Mele, M. Ruiz-Altaba, Z. Phys. C 45, 109 (1989) [Erratum ibid. 47, 676 (1990)].

[5] P. Langacker, Rev. Mod. Phys. 81, 1199 (2009) [arXiv:0801.1345 [hep-ph]].

[6] N. Arkani-Hamed, S. Dimopoulos, G.R. Dvali, Phys. Rev. D 59, 086004 (1999) [arXiv: hep-ph/9807344].

[7] ATLAS Collaboration, ATLAS-CONF-2017-027, 2017.

[8] J. Gunion et al., Phys. Rev. D 40, 1546 (1989).

[9] N. Deshpande et al., Phys. Rev. D 44, 837 (1991).

[10] ATLAS Collaboration, ATLAS-CONF-2016-051, 2016.

[11] N. Arkani-Hamed, S. Dimopoulos, G.R. Dvali, Phys. Lett. B 429, 263 (1998).

[12] P. Baseilhac, V.A. Fateev, Mod. Phys. Lett. A 13, 2807 (1998).

[13] ATLAS Collaboration, ATLAS-CONF-2015-072, 2015.

[14] ATLAS Collaboration, ATLAS-CONF-2016-059, 2016.

[15] ATLAS Collaboration, J. High Energy Phys. 1609, 001 (2016).

[16] ATLAS Collaboration, J. High Energy Phys. 1512, 055 (2015).

[17] ATLAS Collaboration, ATLAS-CONF-2016-055, 2016.

[18] ATLAS Collaboration, ATLAS-CONF-2016-062, 2016.

[19] ATLAS Collaboration, ATLAS-CONF-2016-082, 2016.

[20] https://atlas.web.cern.ch/Atlas/GROUPS/PHYSICS/ CombinedSummaryPlots/EXOTICS/index.html

[21] ATLAS Collaboration, EXOT-2016-21, 2016.

[22] ATLAS Collaboration, ATLAS-CONF-2016-069, 2016.

[23] D. Abercrombie et al., arXiv:1507.00966 [hep-ex].

[24] ATLAS Collaboration, available: https: //twiki.cern.ch/twiki/bin/view/AtlasPublic/ExoticsPublicResults

[25] ATLAS Collaboration, J. High Energy Phys. 1609, 173 (2016). 